\title{
Proposal for a Data Size Saving Office System That Can Be Stored on a Floppy Disk
}

\author{
Miki Sunakawa', Takayuki Fujimoto² \\ ${ }^{1}$ Department of Graduate School of Information Sciences and Arts, Toyo University, Tokyo, Japan \\ s3b101900035@toyo.jp \\ ${ }^{2}$ Department of Graduate School of Information Sciences and Arts, Toyo University, Tokyo, Japan. \\ fujimoto@toyo.jp
}

Article History:Received:11 november 2020; Accepted: 27 December 2020; Published online: 05 April 2021 ABSTRACT : With the development of computer software and applications, various types of recording media
have emerged and evolved. This is a result of the rapid increase in the amount of data created by computers and the
constant demand for large-capacity storage media. As large-capacity recording media have become available at
low prices, the number of content production without being aware of the data capacity is increasing. Rather, content
production often contains unnecessary features and effects for personal use. In this research, we will examine the
feasibility of a practical system by carefully selecting the functions required for general use without excessive
modification of effects. A floppy disk is used as the recording medium to limit the data size of the system.

Keywords: Information Technology, Data size, Data size saving, Recording media

\section{BACKGROUND}

With the development of computer software and applications, various types of recording media have appeared and continue to develop. In particular, the storage capacity is rapidly increasing, and now the data size of one computer is contained in one small recording medium. The evolution of storage media has brought a great impact on improving the convenience and efficiency of our life. Accordingly, the amount of file data generated by computers is rapidly increasing, and this is said to be the result of the constant demand for large-capacity storage media.

Most recording media in the 1980s, when computers began to spread in ordinary households, could store only a small amount of data, which is less than $1 \mathrm{MB}$. Therefore, creators produced content wondering how to decrease the amount of data while being creative. However, in recent years, large-capacity recording media have become available at low prices, and content production is carried out without being conscious of data size as before. In addition, there are many packages of software and applications that contain many unnecessary functions and effects that just increases the amount of data, even though they are used at the individual level. Such a meaningless increase in the data size may slow down the processing speed of the computer or complicate the operation, and may rather reduce the work efficiency and expressiveness of content production.

\section{Purpose}

In this research, we will create the system for content production with small data as possible as it can be. We aim to develop a data size saving office system that is clear to use and easy to operate. By setting a limit on the data size regarding the system production environment in advance, it becomes necessary to carefully select the functions to be installed in the system. It is not just the exclusion of functions, but the thorough selection of functions without impairing the usability. We assume that the user friendliness of the system will be improved and the functionality will be enhanced. In addition, excessive design and beautiful effects, which are often found in modern content, are not always necessary. Regarding systems and applications used at the individual level, they may rather complicate operations and hinder work efficiency. We demonstrate the possibility of a system with which people can handle daily tasks sufficiently without the huge data size unlike today's software and applications'. The system will be established with a simple interface and functions carefully selected only for the general use. We set a limitation: 'a data size saving office application that can be stored on a floppy disk' which means the data size of the system is limited by the recording medium. We plan to make people use the system at the later developmental stage and examine the usability.

\section{CHANGES OF RECORDING MEDIA AND DATA CAPACITY}

This section deals with recording media used in/with computers. There are two types of recording media: a built-in type that is embedded in a computer and a removable type that functions as storage medium and requires

*Corresponding author: Miki Sunakawa

336

Department of Graduate School of Information Sciences and Arts, Toyo University, Tokyo, Japan s3b101900035@toyo.jp 
separate reading device.

A. Transition of prevailing recording media

In the 1970s, through which computers began to be used in some parts of society, cassette tapes for voice recording were used as recording media. Data on Magnetic tapes such as cassette tapes cannot be read randomly. It is necessary to be read from the beginning of the tape each time. However, long-term data storing for several decades is possible if the storage method is correct. Even if the tape is damaged partially, it is rare that the entire data is completely lost. Although it is considered to be a reliable recording medium, it takes time to read the data from the beginning to the end of the tape, so it was replaced by a floppy disk due to lack of the usefulness.

Floppy disks were developed in the 1960s. In the 1980s, when computers became widespread in ordinary households, floppy disks became available at low prices and became the mainstream of data storage. The magnetic disk used for the floppy disk has a structure in which a circular track is divided into sectors at regular angles. Random access to arbitrary data is possible because sectors that contain data have separation. Floppy disks are not suitable for long-term storage of data because it is sensitive to magnetism and humidity. Due to its thin, small and light characteristics, it was widely used as a small recording medium for data exchange and distribution.

In the 1990s, because of the increased data size of systems and software used on computers, there was a gradual shift to recording media that has a larger capacity than floppy disks. Optical discs such as Compact Disc (CD), Digital Versatile Disc (DVD), and Blu-ray Disc (Blu-ray) became widespread. Fine irregularities are carved on the mirror surface on the plastic disk, and the data is read by the difference in the irregularities when the laser beam is applied. Optical discs also have drawbacks such as being vulnerable to scratches, ultraviolet rays, or repeated writing. However, due to the good balance of cost performance and data capacity, many products are still produced and distributed. In addition, optical disc drives can be backward compatible in the order of BD-DVD-CD, and it is also useful in respect of the high versatility of the drive. Besides, Magneto-Optical disk (MO), which is one type of optical disks, also appeared. A magneto-optical memory that reads data by focusing a laser beam on a disk coated with material that emits magnetism when it gets hot. Since the 'disk' is covered with a case, MO disks are resistant to external influences such as magnetism and ultraviolet rays, and are suitable for long-term storage of data. However, it disappeared due to lack of versatility and compatibility like other optical discs.

In the 2000s, the demand for larger-capacity recording media increased than ever before. As a result, Hard Disk Drive (HDD) and semiconductor memories have become smaller and low-priced. The HDD contains multiple magnetic disks (platters) made of aluminum or glass. The magnetic head reciprocates at high speed on rotated platters to read the data. Although high-speed access to data is possible, it is vulnerable to external stimuli and data corruption or loss is likely to occur. On the other hand, semiconductor memory does not require a rotation mechanism, whereas data on many types of recording media is read with a rotation operation. Semiconductor devices enable ultra-high-speed access to data by semiconductor element without physical limits. There are also Universal Serial Bus (USB) and Secure Digital Memory Card (SD memory card) which are non-volatile flash memories that do not lose data for a long time even if there is no power supply. Thus, in recent years, recording media by semiconductor memory have come to be widely used. On the other hand, it is still underway for further development because the unit price per storage capacity is expensive and the number of rewrites of semiconductor elements is limited.

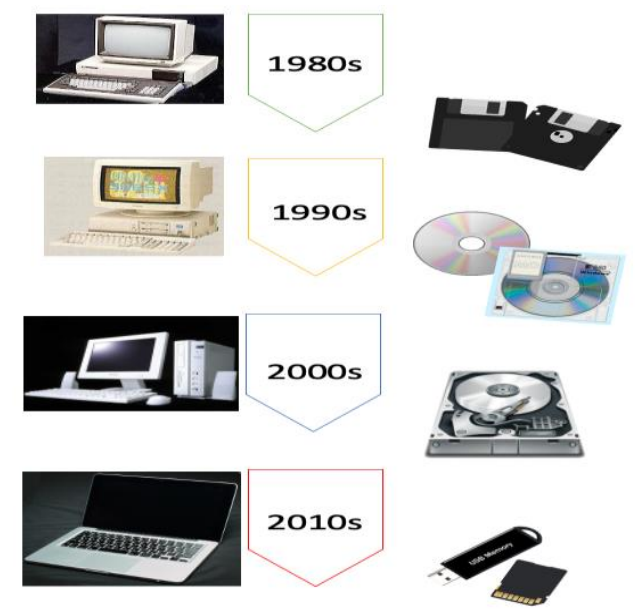

Fig 1. Advances of Computers and Storage Media

\section{B. Increase of data capacity}

From the transition of the data capacity that can be stored in the recording media that have been used for computers, it is clear that it has made a dramatic evolution in just about 50 years. 


\begin{tabular}{|c|c|c|c|}
\hline & Storage device & Age of use & Data capacity (approximate) \\
\hline Cassette tape & Magnetic tape & 1970 s & $520 \mathrm{~KB} 2 \mathrm{MB}$ \\
\hline Floppy disk & Magnetic disk & $1980 \mathrm{~s}$ & $80 \mathrm{~KB} \sim 1.44 \mathrm{MB}$ \\
\hline$C D$ & Optical disk & 1990s Present & $210 \mathrm{MB} \sim 700 \mathrm{MB}$ \\
\hline HDD & Magnetic disk & Late 1990 s Present & 20TB \\
\hline SSD & Semiconductor memory & 2000s Present & $\sim 60 \mathrm{~TB}$ \\
\hline
\end{tabular}

Table 1. Comparison of storage media

The maximum data capacity of cassette tapes used as recording media for computers was about 2MB. This data capacity cannot store data that deserves just one piece of photo taken with the current smartphone. However, the content handled by computers at that time was several hundred KB at maximum, and the cassette tape data capacity was enough. Currently, HDDs widely distributed as major recording media, includes products that have data capacity of 20TB. 20TB is about 10 million times the size of the cassette tape data capacity.

Floppy disks have become widespread as recording media that can be easily handled for data storage, but they were once distributed as the Operating System (OS) for computers. However, the data size of current OS cannot be stored on a floppy disk. Instead, it is recorded on subsequent recording media that can store a large amount of data, such as CD-ROM (Compact Disc Read only memory) and USB. Other systems and applications are also undergoing similar changes. Major recording media are shifting from floppy disks to the products that have enormous data capacities; optical disks, HDDs, and semiconductor memories. In short, it can be said that the evolution of recording media is always related to the improvement of computer performance and the increase in data size of digital content.

Such increase is greatly influenced by the fact that digital content has been made multifunctional in order to improve users' convenience. However, it often contains features that are not used at the individual level. On the contrary, there is a possibility that the variety of functions lead to complicated settings and operations. In addition, as the performance of recording media improves, elaborately created decorations and layout designs for content is increasing. Beautiful design is preferred by people, but this is a major factor to increase data size. Beautiful design may cause negative effect: it may take a long time to read the design data, or how to operate may be unclear.

\section{DEVELOPMENT OF DATA SIZE SAVING SYSTEM}

\section{A. The production environment}

In this research, we work on the production of a data-saving system that can be used on a daily basis and requires a small amount of data capacity. It is necessary to set the particular data capacity that represents "data-saving" for the production. Therefore, a floppy disk is adopted as a recording medium for storing the created system.

Because of the nature of developing a data-saving system that anyone can easily use at the individual level, it is desirable to carry out development in an environment that can be easily arranged by a general method. Although production of floppy disks is ended, they are still on the market in large numbers. Floppy disk is still a relatively easily available product in this respect. The same is true for floppy disk drives needed to read and write data. The development of a data-saving system using a floppy disk is suitable because the system that operates without problems in the current computer environment and occupies the data capacity as small as possible.

\section{B. Floppy disk}

A floppy disk is a magnetic disk stored in a thin card-shaped case. There are three types: 8 inch, $5.25 \mathrm{inch}$, and 3.5 inch. From the 1980 s to the early 1990s, it was used as the leading recording medium globally. It was replaced by subsequent recording media and production was discontinued around 2011. Therefore, the number of young people who do not know the existence of floppy disks is increasing. The most commonly distributed was the type 3.5 inch, which had a storage capacity of 1.44MB. This data capacity cannot afford even one file regarding a word file or PowerPoint file with effects created in the current system environment. Today's USB memory and flash memory have a storage capacity of TB size, and many of them are smaller than floppy disks in respect of size. Therefore, the social mission of floppy disks has almost disappeared, except the case in which it is unavoidable to use some old systems.

However, the data capacity of the 3.5 inch floppy disk, $1.44 \mathrm{MB}$, can store about 1.44 million single-byte characters for text data. It is possible to record data of a number of characters that deserves several books or more. In other words, the floppy disk has the sufficient data capacity for the number of characters used in our daily writings. At least, for document management, floppy disks are still useful today in respect of the capacity. The reason why the floppy disk cannot store even one file of current standard, is because the text files created by word processing software or PDF are not only character data, but also the bloated data due to the added various modified effects and formatting of the file. 


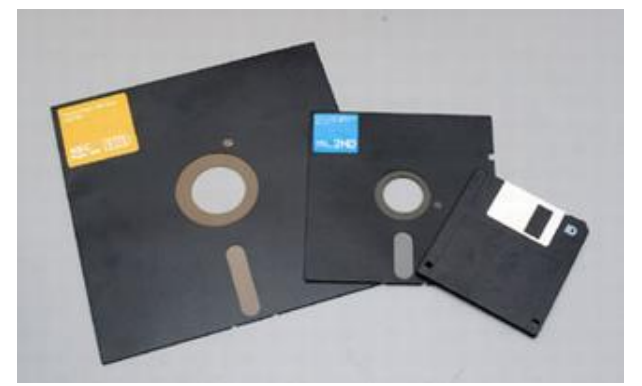

Fig 2. Floppy disks (8 inch, 5.25 inch, 3 inch from the left)

\section{Production with floppy disk}

Floppy disks were the main recording medium from the 1980s to the early 1990s. Creators used floppy disks to store the content that they created. It was necessary to care for the amount of content data so that it fits in the data capacity of the floppy disk. For example, regarding music in the market that was on a floppy disk for storage, it was set as music software that has only 1 to 3 songs. To store music data on floppy disks, it is necessary to change the data format and thoroughly compress the data. Even so, there is no problem with listening because the minimum sound quality is maintained. There are some musicians who dare to adopt the floppy disk to store music for sale, and their compressed music data has been re-evaluated in respect of the unique sound quality.

Other than music, video games are a typical example of floppy disk content that have been widely distributed. In 1983, "Disk System" that incorporated the characteristics of floppy disks into the Nintendo (NES) Family Computer: the world-famous home video game device, appeared. It was necessary to create a video game according to the data capacity of the disk system. Specifically speaking, the game content needed to be carefully devised for storage. The graphic data was reused in the same game, and the data was compressed to the extent that the graphics did not collapse. Various efforts have been made to reduce the amount of data to the utmost limit. As a result, the data size of the world's best-selling video game "Super Mario Brothers" was only 40KB.

In the past, efforts have been made to produce high-quality digital content while trying reducing data size in order to record all the content in small data capacity. According to the limited data capacity at that time, the creators made various efforts to compress the data and to be creative with expression without degrading the content quality. Under such circumstances, the creativity of the creators was demonstrated, and many of the content at that time can be still sufficiently enjoyable now.

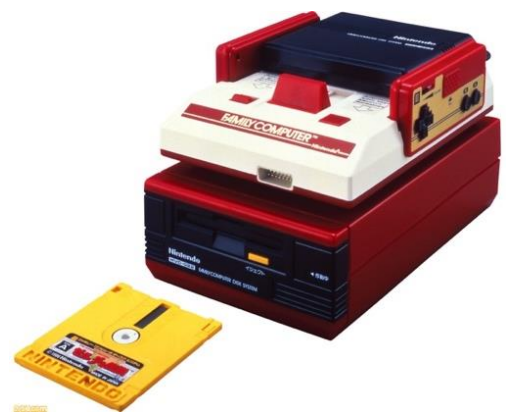

Fig 3. Nintendo "Quick Disk" device and game software

\section{SYSTEM PROTOTYPE}

In this research, we create a system using a floppy disk based on the concept of being creative with expression regarding the data capacity as small as possible. This research aims to 'data-saving in content production' that is discounted than before with the device development and the advent of large-capacity recording media. It does not mean simply limiting functionality to reduce data size. We make the system simple and easy-to-understand visually while having practical functions. A 3.5 inch floppy disk is the most popular size as the floppy disk. Even in the current computer usage environment, it is easy to use casually in respect of size and thickness. As a data-saving application system that can be stored on a 3.5 inch floppy disk that has $1.44 \mathrm{MB}$ data capacity, we develop four applications as explained in the next chapter. The development language is mainly a combination of JavaScript and HTML.

\footnotetext{
${ }^{*}$ Calculator (calculation)

* Text editor (document creation)

* Paint software (design)
} 
* Video player (media player)

\section{A. Calculator}

Calculator is one of the functions that are often used in daily life regarding the use of computers. It is no exaggeration to say that the four arithmetic operations are always used out of the basic functions. In addition, there are many opportunities to use the operations on decimals, which is often used in tax rate calculations. Therefore, the calculator in this system specializes in the functions of four arithmetic operations and the decimal function as a simple and highly practical combination. In consideration of operability, the buttons are set large by default and a screen layout that visualizes the calculation progress is adopted.

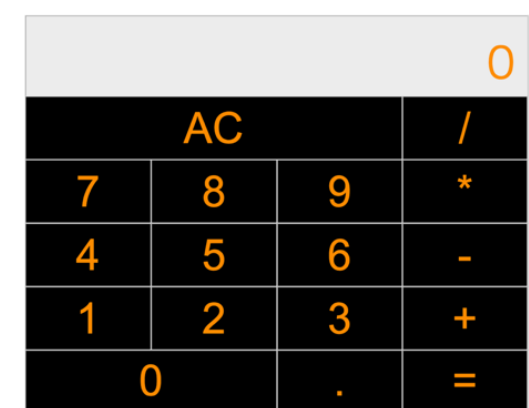

Fig 4. screen of the prototype text editor

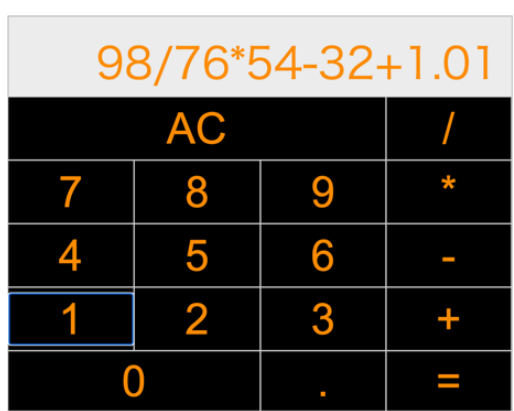

\section{B. Text editor}

Fig 5. Input screen of the prototype calculator

In general computer use, one of the most frequently used software is word processing software. It is no exaggeration to say that document creation is a core function of computers. Recent word processing software has rich functionality and design variations, and the data size in a document file is extremely large. This is because layout and block copy data are incorporated other than character data, as a result of the people's pursuing convenience and appearance regarding the document. Needless to say, if effects are applied besides them, the amount of data will increase more.

However, in document creation, extra effects and layout often reduce work efficiency. Rather, the simple appearance and functions make the operations easier to understand, and it may lead to better work efficiency and convenience. Therefore, in this research, we intentionally eliminated unnecessary effect functions and implemented a very simple text editor. Document creation functions are limited to character input and deletion, and saving the completed texts. In addition, the system has only the minimum necessary layout as far as it can smoothly handle document creation.

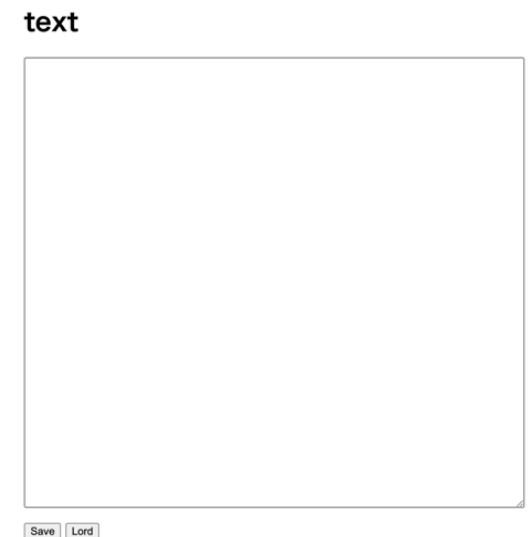


Fig 6. screen of the prototype text editor

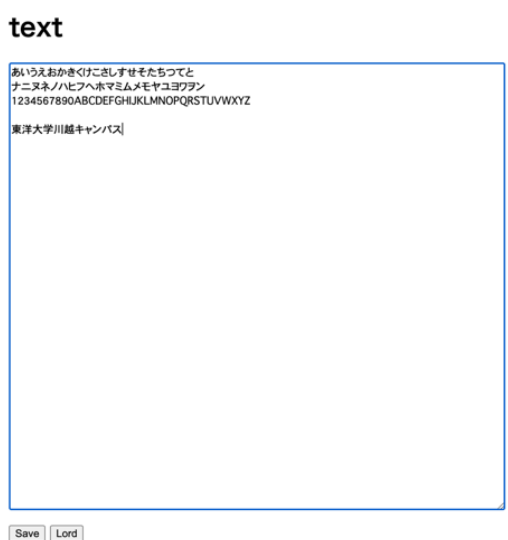

Fig 7. Input screen of the prototype text editor

\section{Paint software}

The paint software is created assuming a simple one that can be operated with a mouse or touch-pad. As the minimum functions for painting, we selected changing the color, changing the thickness of the line, and deleting what was drawn. In particular, the color change function will be implemented so that fine adjustments can be made because each user tends to have each color preference in the drawing work. The functions of paint software can be fully understood even just with a quite simple appearance compared to ones of other systems. Therefore, the system is implemented with the minimum necessary layout that mainly features the drawing space.

\section{paint $\Leftrightarrow$}

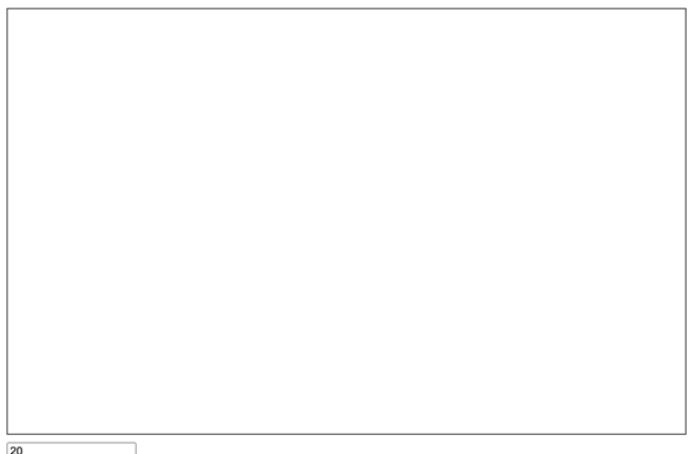

Fig 8. Screen of the prototype paint software

\section{paint $\Leftrightarrow$}

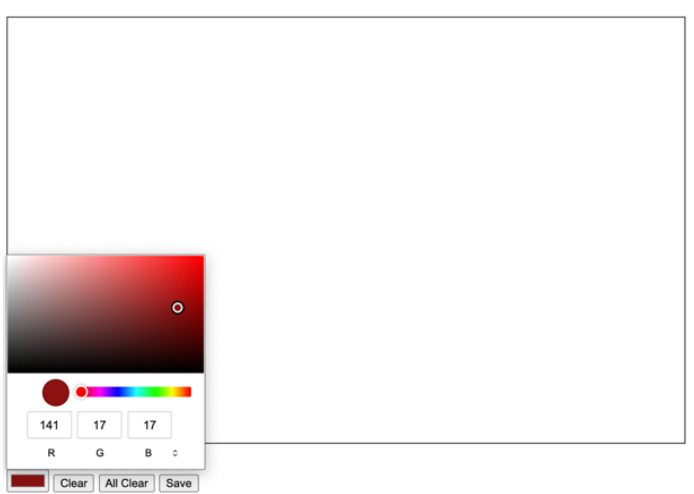

Fig 9. Color selection of the prototype paint software

\section{Video player}

Many kinds of the video player software for computers have not only 'play' functions but also 'edit' functions. The video player in this system focuses and produces only the functions related to 'play'. It has basic 'play' functions; 'start', 'pause' and 'volume control' buttons to play the video. It also has a button to access to the start or 
end points of the video, and buttons for rewinding and forwarding in a second. The playpoint can be changed not only by clicking the button but also by moving playhead on the play bar, which is a conventional operation method. In addition, a button to set the play speed is also introduced because the style of watching videos at double the speed is increasing accompanied with the spread of video sharing sites. Since the name of the function is represented on each button, we suppose that the function can be fully understood just with simple display.

Regarding playing videos on a computer, it is becoming more common to watch videos while doing some other tasks at the same time. This is the so-called 'background mode' as to the viewing style. Therefore, the screen display mode can be changed. It is possible to play the video in the small window (background) mode, which makes it easy to work such as document creation while playback. They system also has the full-screen display mode that allows users to appreciate the details of the part that you are interested in.

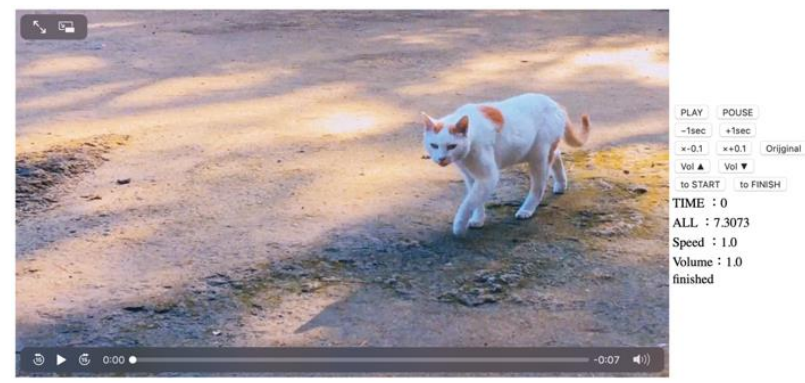

Fig 10. Playback screen of the prototype video player

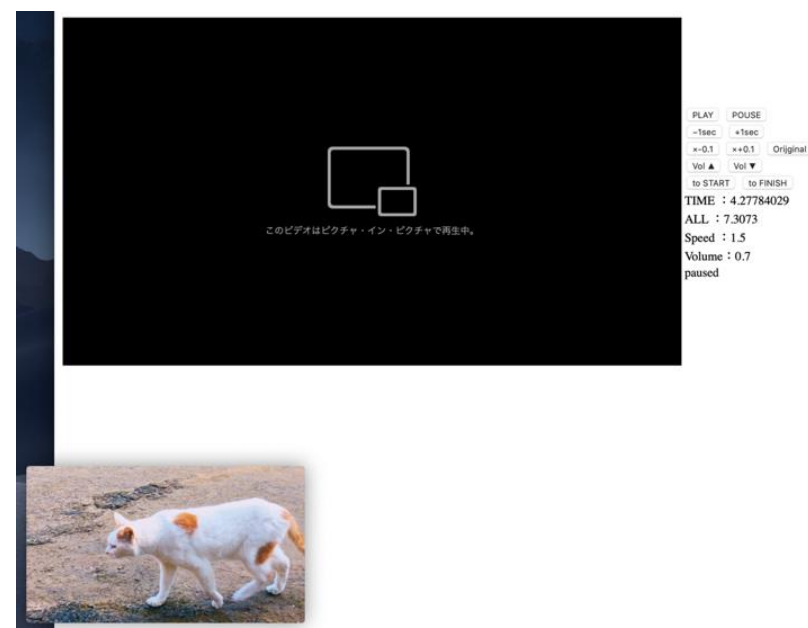

Fig 11. Playback screen in a small window

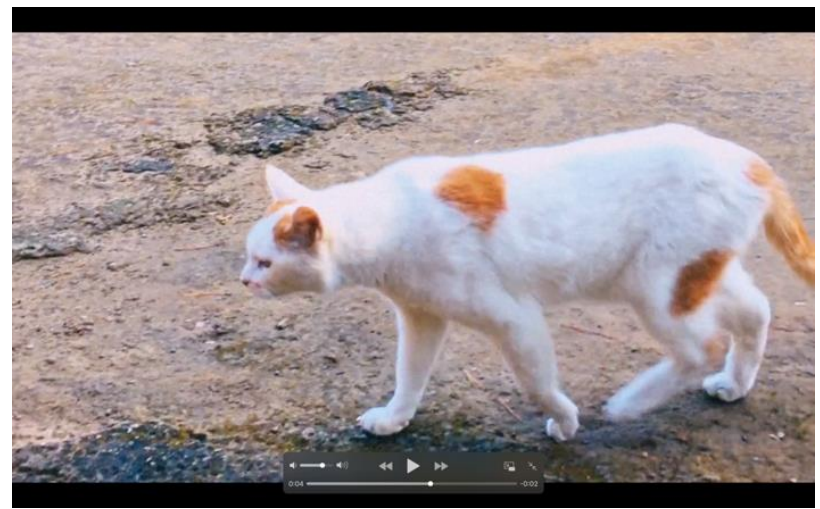

Fig 12. Playback in full screen mode

\section{FUTURE WORK}

Around the 1980s for which computers gradually became widespread in ordinary households, most recording media had small data capacity of less than 1MB. The data capacity worked well because the systems and applications in use at that time and the data size of the files created with them was extremely small. In addition, in order to fit the systems in a recording medium that has a small data capacity, the creators created the content making effort to be creative with expression while saving the amount of data, by trial and error. However, in recent 
years, large-capacity recording media have become available at low cost. It is often the case that content production is carried out without being conscious of the amount of data as before, and developmental efforts to cope with the data capacity have low priority now. Relatedly, content often has functions and modification effects that are not always necessary for general use. The wide variety of functions and beautiful layout tend to be overrated. However, the question remains regarding the current situation where systems and applications with such a huge data are the mainstream.

In this research, we created the office system that can be stored on a floppy disk with a capacity of $1.44 \mathrm{MB}$ with data reduction by carefully selecting the minimally necessary functions and interfaces. We have prototyped four types of applications: a calculator, a text editor, painting software and a video player. All have very simple functions and screen layout. The prototype system was created with consideration of usability and visibility, but it is necessary to improve the functions and design by the actual use with the subject experiment. Although currently the four types of applications are configured, additionally, we would like to prototype new systems as far as they can be stored in 1.44MB. Eventually, the system of the proposal will be completed as one 'data size saving office application that can be stored on a floppy disk' consisting of multiple systems, and the overall usability will be examined.

\section{ACKNOWLEDGMENT}

This work was supported in part by the U.S. Department of Commerce under Grant BS123456.

\section{REFERENCES}

Information Processing Society of Japan, "Japanese Computer Personal Computer History of Birth and Development", Information Processing Society of Japan Computer Museum, http://museum.ipsj.or.jp/computer/personal/history.html (2020/7/4).

Humming Heads Inc., "History and classification of recording media", 2014/7/24, https://www.hummingheads.co.jp/reports/feature/1407/140724_01.html\#1 (2020/7/4).

SONY Corporation, "How Blu-ray Disc works", https://www.sony.jp/bd/about/technology/index.html (2020/7/4).

Fujitsu, "Storage technology terminology", https://www.fujitsu.com/jp/products/computing/storage/lib-f/tech/ (2020/7/4).

BUFFALO Inc., "What is SSD? How is it different from HDD?", https://www.buffalo.jp/topics/select/detail/124105699_1523.html (2020/7/4).

THE VERGE, "US nuclear force will phase out floppy disks next year", 2016/5/16, https://www.theverge.com/2016/5/26/11782716/pentagon-nuclear-force-floppy-disk-outdated-tech (2020/7/4).

Neowin, "Report-US-government-spends-tens-of-billions-on-maintaining-50-year-old-computer-systems", 2016/5/25,

https://www.neowin.net/news/report-us-government-spends-tens-of-billions-on-maintaining-50-year-old-co mputer-systems (2020/7/4).

Nintendo Co., Ltd., “That secret that will be revealed now! What was the 'disk system'?”, Nintendo Online Magazine, 2004/8 No.73, https://www.nintendo.co.jp/nom/0408/index.html (2020/10/5).

Gabriela Helfet, "Techno producer Remute releasing new album on floppy disk", The Vinyl Factory, 2017/11/6, https://thevinylfactory.com/news/remute-new-album-floppy-disk/ (2020/10/5).

Christopher R. Weingarten, "Revolution at 3.5": Inside Vaporwave's Mini-Boom of Floppy Disk Releases", Rolling Stone, 2018/7/2, https://www.rollingstone.com/pro/features/vaporwave-floppy-disk-trend-666085/ (2020/10/10).

Gareth Corfield, "Pen Test Partners: Boeing 747s receive critical software updates over 3.5" floppy disks", The Register,

2020/8/10, https://www.theregister.com/2020/08/10/boeing_747_floppy_drive_updates_walkthrough/ (2020/11/3).

Japan Electronics and Information Technology Industries Association, "CD / DVD / Blu-ray Disc Q \& A collection”, https://home.jeita.or.jp/cgi-bin/page/detail.cgi?n=233\&ca=14\#type (2021/1/3).

Business Wire, "Seagate Accelerates Enterprise Momentum With Two New Flash Products", 2016/8/9, https://www.businesswire.com/news/home/20160809005479/en/Seagate-Accelerates-Enterprise-Momentu m-Flash-Products (2021/1/3).

Nikkei Business Publications, Inc., Nikkei XTECH Techno Trend beyond 2020 "HDD over 50TB in the latter half of the 2020s, supporting the information explosion era", 2019/12/26, https://xtech.nikkei.com/atcl/nxt/column/18/01140/00002/ (2021/1/4).

ASAHI INTERACTIVE, Inc., CNET Japan "The world seen by smartphone natives for teens, "double speed viewing" is the norm for videos-- "cost performance" is important for time", 2020/1/25, https://japan.cnet.com/article/35148371/

(2021/1/4). 
